\title{
Clinical management of a challenging malignancy, osteoblastoma-like osteosarcoma: a report of four cases and a review of the literature
}

\author{
This article was published in the following Dove Press journal: \\ Therapeutics and Clinical Risk Management \\ 17 August 2016 \\ Number of times this article has been viewed
}

\author{
Harzem Ozger' \\ Bugra Alpan' \\ Mehmet Salih Söylemez ${ }^{2}$ \\ Korhan Ozkan ${ }^{3}$ \\ Ahmet Salduz ${ }^{4}$ \\ Bilge Bilgic ${ }^{5}$ \\ Basak Kumbasar Sirin ${ }^{6}$ \\ 'Department of Orthopaedics and \\ Traumatology, Maslak Acibadem \\ Hospital, Istanbul, ${ }^{2}$ Department of \\ Orthopaedics and Traumatology, \\ Bingöl State Hospital, Bingöl, \\ ${ }^{3}$ Department of Orthopaedics and \\ Traumatology, Faculty of Medicine, \\ Istanbul Medeniyet University, \\ ${ }^{4}$ Department of Orthopaedics \\ and Traumatology, ${ }^{5}$ Department \\ of Pathology, Faculty of Medicine, \\ Istanbul University, ${ }^{6}$ Department of \\ Radiology, Maslak Acıbadem Hospital, \\ Istanbul, Turkey
}

\begin{abstract}
Osteoblastoma-like osteosarcoma, a rare form of osteosarcoma, is a malignant lesion associated with risks of both local recurrence and distant metastasis. The differential diagnosis of osteoblastoma-like osteosarcoma from osteoblastoma and aggressive osteoblastoma remains controversial and challenging. Previous studies suggest that these three types of tumor are distinct entities. However, working out a precise diagnosis may not always be possible immediately on the basis of initial clinical, radiological, and histopathological features. On the other hand, the importance of a correct diagnosis cannot be overemphasized since the treatment strategies change dramatically according to the nature of the lesion. In all of our cases, initial Tru-Cut biopsies revealed osteoblastic features with minimal atypia, but further biopsies confirmed malignancy. A high index of suspicion and considerable experience are prerequisites for accurate diagnosis in case of clinicopathological and radiological discordance.
\end{abstract}

Keywords: osteoblastoma-like osteosarcoma, differential diagnosis, biopsy

\section{Introduction}

Osteoblastoma-like osteosarcomas (OBLOSs) constitute 1.1\% of all osteosarcomas. OBLOS shares similar histopathological characteristics with osteoblastoma, which is a benign primary bone-forming tumor. ${ }^{1}$ However, OBLOS is a malignant lesion, and without appropriate treatment, it has a high risk of mortality.

The identification and management of borderline cases of osteoblastoma, aggressive osteoblastoma, and OBLOS remain problematic. Theoretical knowledge suggests that these three types of tumor are different entities. ${ }^{2,3}$ Osteoblastoma is considered as a stage 2 (active) benign tumor, whereas aggressive osteoblastoma is designated as a stage 3 (aggressive) benign tumor. Aggressive osteoblastoma has a higher growth potential than conventional osteoblastoma and also has a higher risk of local recurrence. ${ }^{4}$ OBLOS, a rare form of osteosarcoma, is a malignant lesion associated with risks of recurrence and metastasis. ${ }^{2}$ The differentiation of osteoblastoma from OBLOS on the basis of pathological features alone is a major challenge. Despite the deceptive nature of histopathology, there is no room for diagnostic inaccuracy since the treatment strategy differs markedly for OBLOS and an error in judgment will result in either unnecessary morbidity or compromised local and systemic tumor control. ${ }^{5}$

In this study, we describe four cases of OBLOS and emphasize the importance of differential diagnosis and selection of appropriate treatment. The Institutional Review Board of Acibadem University, Faculty of Medicine deemed it unnecessary to obtain ethics approval for this case series because treatment modalities performed
Correspondence: Mehmet Salih Söylemez Department of Orthopaedics and Traumatology, Bingöl State Hospital, Kültür Mahallesi, Oguzhan Caddesi No 22, 12000 Bingöl, Turkey Email slhsylmz@gmail.com 
in this study were accepted by the patients. Written informed consent was obtained from all patients for treatment, as well as for publication of de-identified data and medical images. This study complied with the guidelines from the Declaration of Helsinki.

\section{Case I}

A 6-year-old girl presented to our tumor clinic with increasing swelling and pain on the dorsum of the hand and around the wrist for the previous 3 months. She had no history of trauma. Physical examination of the wrist revealed exquisite tenderness over the radial styloid. No palpable mass or erythema was present over the wrist. She had almost full range of motion, and a neurovascular examination yielded normal results. Laboratory values were also within normal limits. Radiography revealed an eccentrically located lytic expansile lesion with permeative margins in the distal metaphysis of the radius. The cortex appeared to be thinned, suggestive of a periosteal reaction (Figure 1). Magnetic resonance imaging (MRI) demonstrated central sclerosis and heterogeneous enhancement. Whole-body (99m)-technetium (Tc) bone scintigraphy revealed increased uptake by the distal radial metaphysis.

Osteosarcoma, aggressive osteoblastoma, Ewing's sarcoma, and osteomyelitis were considered in differential diagnosis. Tru-Cut biopsy was performed. The sections revealed the presence of an active osteoblastic lesion. Immature osteoid formed trabecular woven bone that was generally well organized but interconnected in a random manner in some places. Neither osteosarcoma nor malignancy could be confirmed with histopathological findings. However, due to the presence of immature osteoid production and possibility of malignancy according to radiology, the senior author and the musculoskeletal pathologist agreed to go on with an

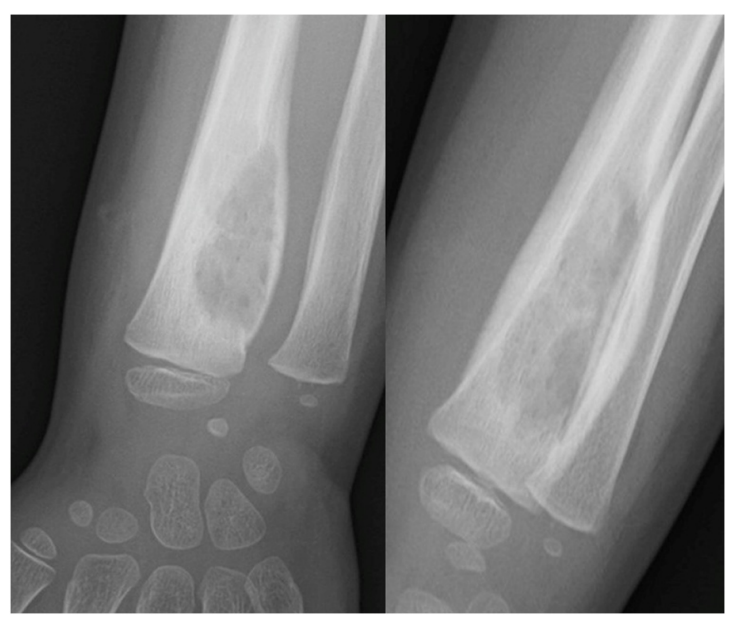

Figure I X-rays showing a lytic expansile lesion with permeative margins in the distal metaphysis of the radius at initial admission.

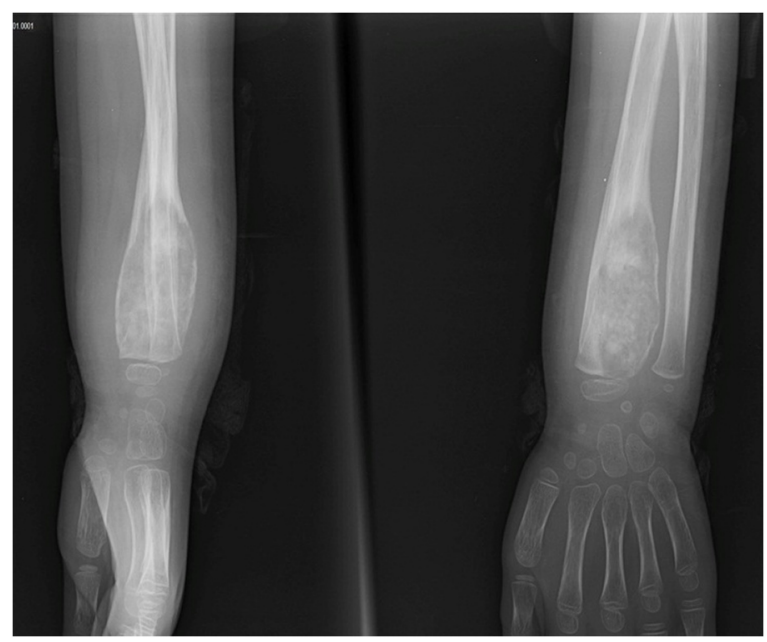

Figure 2 X-rays showing tumor progression after the second biopsy.

open biopsy 15 days after the first biopsy to permit precise differential diagnosis.

Trabecular bone formation, osteoclastic giant cells, and, in some areas, epithelioid osteoblasts were evident in biopsy sections. The examination of the second biopsy also resulted in favor of osteoblastoma. The patient was kept under close observation.

Twenty-five days after the second biopsy, the patient presented with progressive tenderness and pain. Follow-up radiographs of the wrist revealed tumor progression (Figure 2).

An additional open biopsy was performed, and sections from all three biopsies were evaluated comparatively. This evaluation revealed a neoplasm with variable morphology. Sections from the third biopsy showed trabecular bone surrounded by osteoblasts and osteoclasts, pleomorphic cell penetration, and randomly organized immature osteoid (Figure 3). The latest pathological findings in the light of clinical and radiological changes led to the diagnosis of OBLOS.

The patient underwent neoadjuvant chemotherapy. After completion of therapy ( 5 months after presentation), the distal radius was removed en bloc with wide margins, and the distal ulna was transposed to the radius. The resected specimen revealed $95 \%$ necrosis of the residual tumor. The patient underwent three additional courses of adjuvant chemotherapy. At the time of last follow-up (26 months postoperatively), no evidence of recurrence or metastasis was observed.

\section{Case 2}

A 27-year-old male presented to our clinic complaining of pain in his right shoulder. The pain was constant but did not 

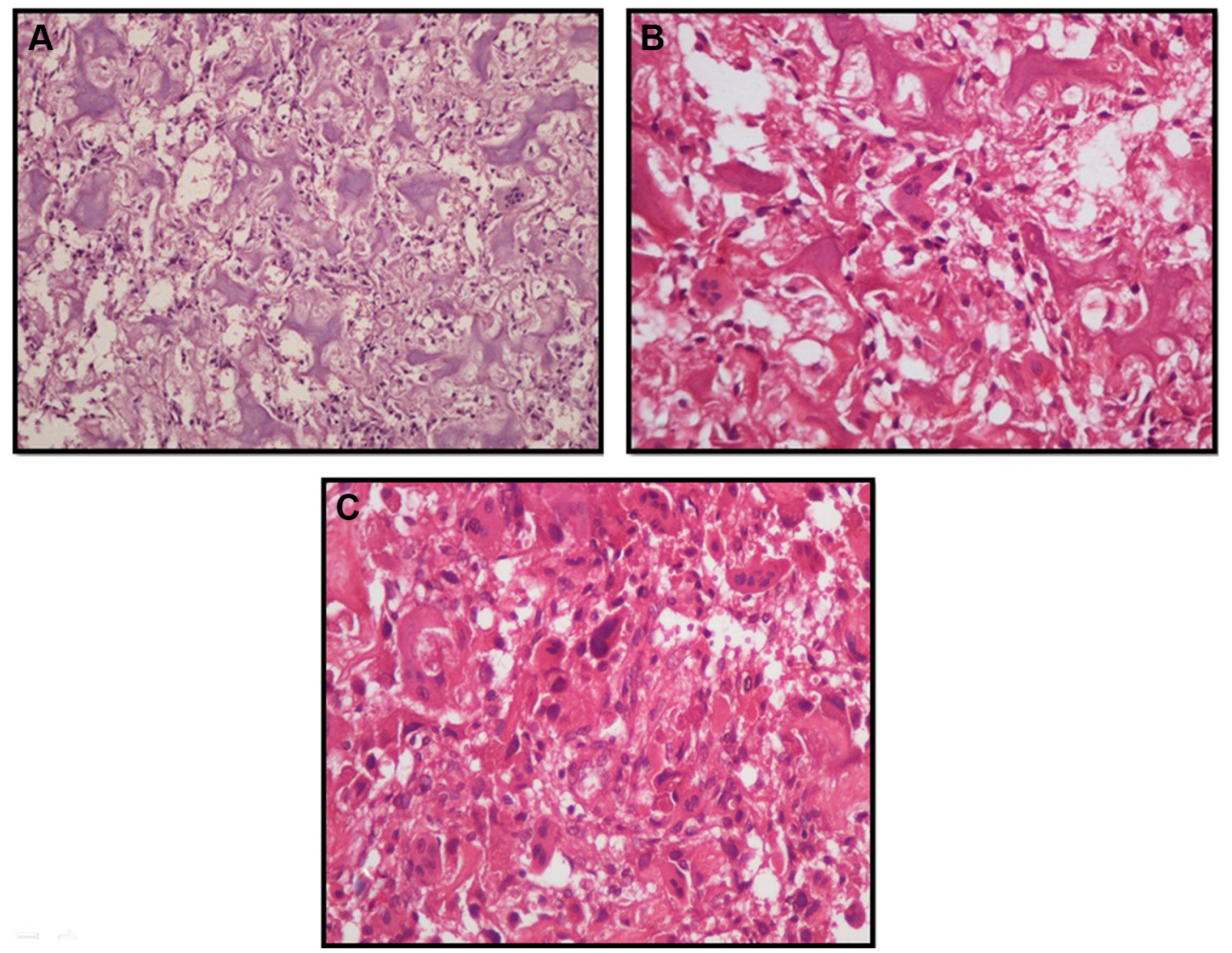

Figure 3 H\&E staining.

Notes: $(\mathbf{A}) \times 100$ and $(\mathbf{B}) \times 200$. Both images reveal somewhat irregular osteoid production and preserved trabecular architecture. Osteoblastic rimming and scattered osteoclasts are evident, separated by minimal atypia. (C) $\times 400$. Focal pleomorphism can be observed.

Abbreviation: H\&E, hematoxylin and eosin.

compromise his range of motion. Preoperative radiographs and computed tomography (CT) of the humerus revealed a lytic-sclerotic cortical lesion located at the lesser tubercle. The lesion was $\sim 1 \mathrm{~cm}$ in diameter, and nidus formation was also apparent on CT (Figure 4A1 and A2). Preoperative MRI revealed central sclerosis and edema of the surrounding bone. No cortical expansion or soft-tissue lesion was evident (Figure 4B1 and B2). Whole-body Tc-99 bone scintigraphy revealed increased uptake by the lesser tubercle of the right proximal humerus (Figure 4C).

The lesion was excised with curette and high-speed burr with the radiological diagnosis of osteoid osteoma/ osteoblastoma. The pathology was reported as "benign osteoblastic tumor". However, the symptoms recurred after 2 months. A limited curettage was performed in the form of incisional biopsy. The pathological specimen again exhibited features consistent with osteoblastoma with epithelioid cells lining the trabeculae in focal areas. However, such an early local recurrence was found to be suspicious for a benign osteoblastic tumor, and a new Tc-99 bone scan was performed. This new whole-body scan revealed increased uptake in the right proximal humerus, as expected due to recent recurrence and surgical intervention and also in the lateral border of the body of left scapula, to the authors' surprise (Figure 5). Excisional biopsy with wide margins was performed for the scapular lesion, and the result of the pathology was consistent with OBLOS. The surgical margins were negative. Chemotherapy was initiated.

Follow-up MRI after neoadjuvant chemotherapy revealed possible intraarticular and periarticular involvement of the right shoulder joint. Wide resection of proximal humerus including extraarticular resection of the glenohumeral joint was performed, and the defect was reconstructed with tumor prosthesis. The pathological specimen from the right proximal humerus was consistent with OBLOS (Figure 6). The tumor necrosis rate was $90 \%$. At 23 months postoperatively, the patient was alive with no local recurrence or metastasis.

\section{Case 3}

A 15-year-old male patient presented with swelling and limited range of motion in his right wrist. His parents reported no history of trauma. Although the volar aspect of the distal 

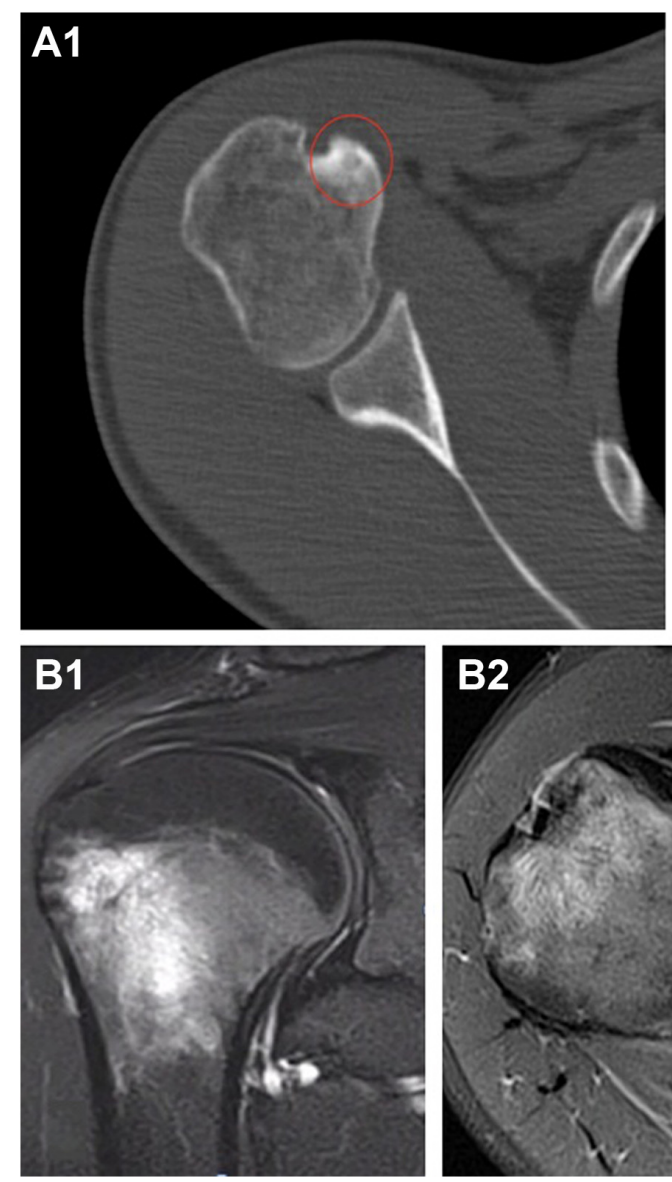

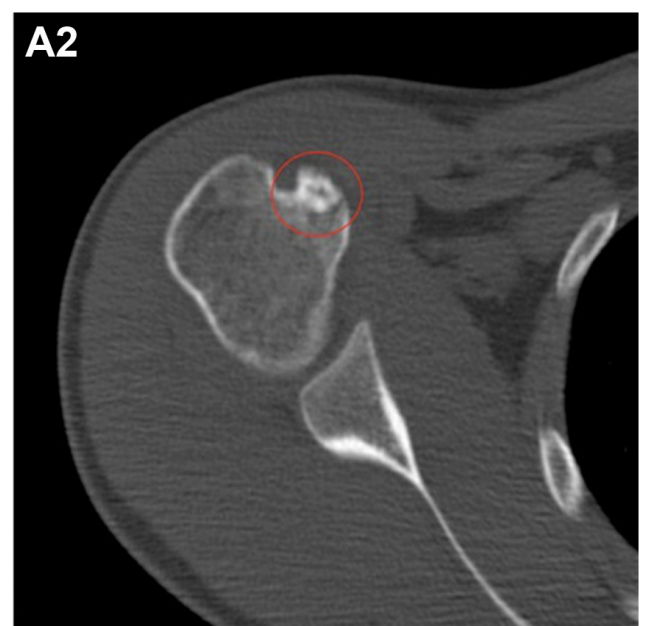

C

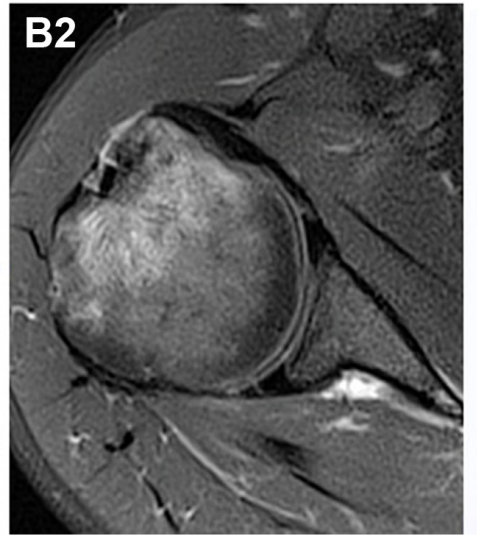

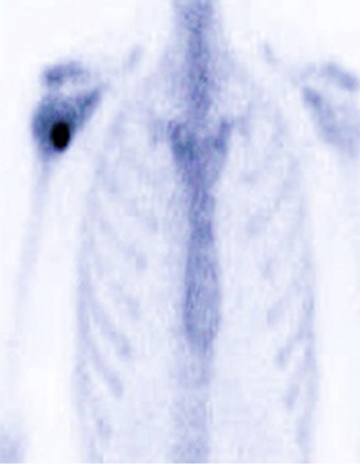

Figure 4 Preoperative images obtained by CT (AI, A2), MRI (BI, B2), and bone scintigraphy (C).

Note: Red circles are showing nidus formation on $\mathrm{CT}$ images.

Abbreviations: $\mathrm{CT}$, computed tomography; MRI, magnetic resonance imaging.
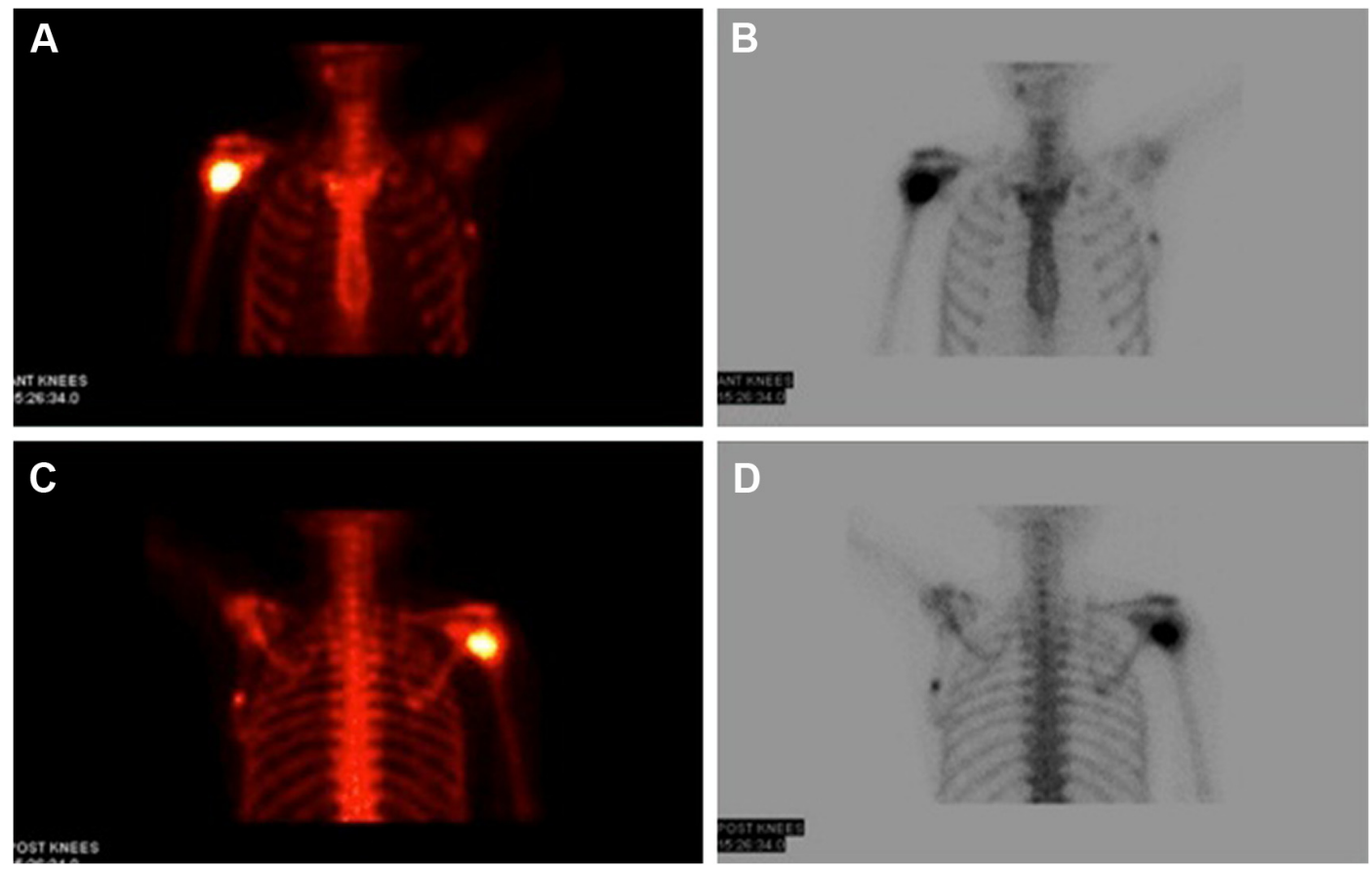

Figure 5 Anterior-posterior (A, B) and posterior-anterior (C, D) technetium- $(99 \mathrm{~m})$ bone scintigraphy showing increased uptake by the right proximal humerus and the lateral border of the left scapula. 

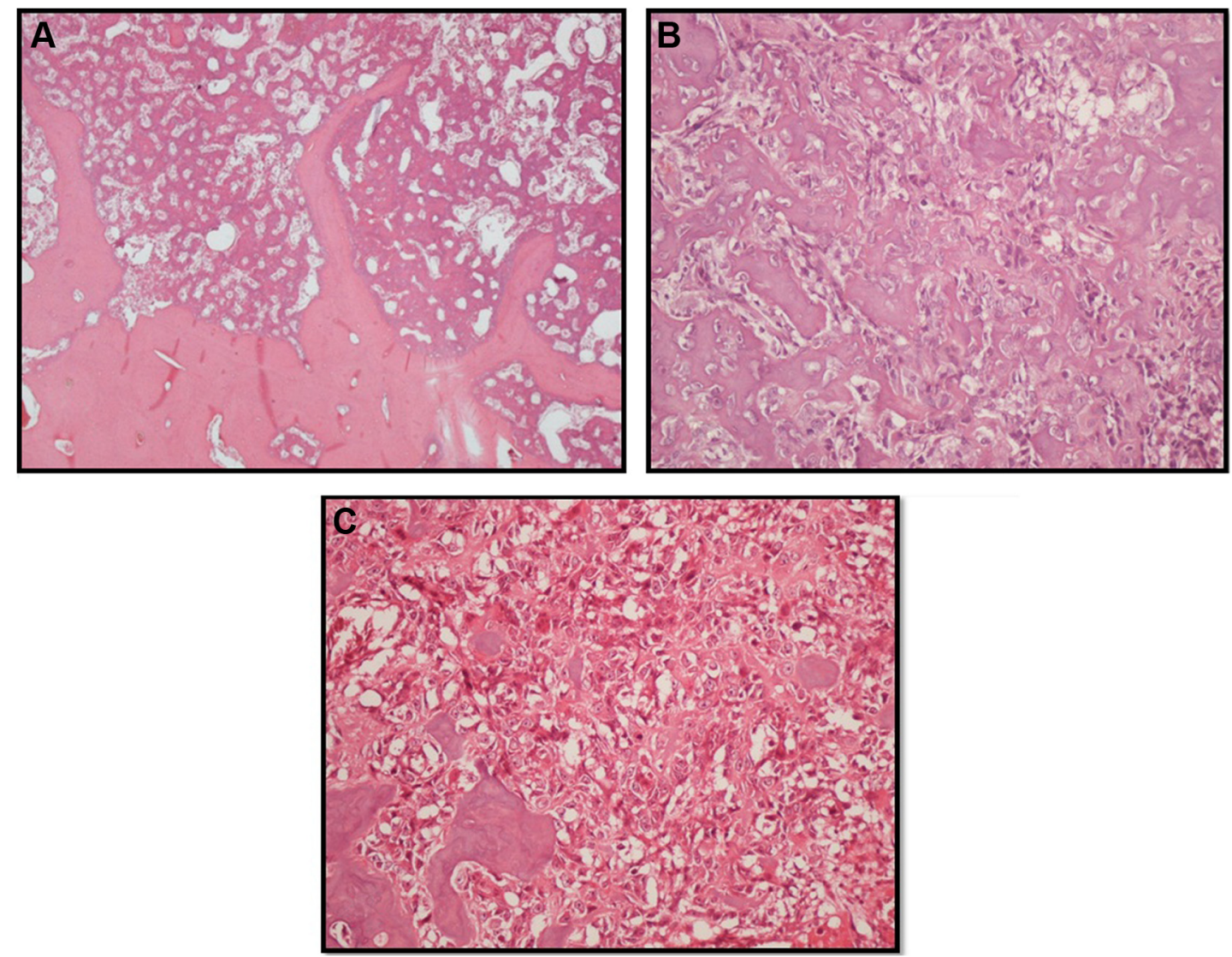

Figure 6 H\&E staining.

Notes: $(\mathbf{A}) \times 100,(B) \times 200$, and $(\mathbf{C}) \times 200$. (A and B) (Right humerus): (A) prominent osteoid matrix with minimal cellular component and without obvious pleomorphism is observed. (B) An osteoblastoma-like bone formation is evident, but host bone entrapment reveals the infiltrative nature of the tumor. (C) (Left scapula) "Nidus"-like cellular focus surrounded by dense, hypocellular osteoid is shown.

Abbreviation: H\&E, hematoxylin and eosin.

radius was tender with deep palpation, no sign of local inflammation was evident. Neurovascular examination was unremarkable. Anteroposterior radiograph of the right wrist revealed a focal area of mixed radiolucency and irregular opacity in the central part of epiphysometaphyseal region (Figure 7A). CT and MRI better demonstrated the eccentric, lytic, minimally expansile lesion with obvious periosteal reaction and permeative margins in the metaphysis, extending into the epiphysis (Figure 7B1 and B2, C1 and C2). Marked soft tissue enhancement was observed in the volar aspect of lesion. Central sclerosis was absent on MRI.

A Tru-Cut biopsy was performed. The sections demonstrated trabecular bone formation surrounded by osteoblasts and osteoclasts. Atypia was not obvious. Focal areas of lacelike osteoid were noted; a definitive diagnosis could not be established. The case was consulted with an international reference center in orthopedic oncology, including the clinical and radiological findings and pathology slides of the original biopsy. The lytic appearance at the periphery of the tumor in radiographs and CT, the pattern of osteoid formation, and the presence of host bone permeation in some areas in histology were regarded as adequate evidence to establish a diagnosis of OBLOS (Figure 8).

The patient underwent neoadjuvant chemotherapy. After completion of therapy, intraarticular wide resection of the distal radius and reconstruction with vascularized fibula (including the proximal epiphysis) to the radius were performed. The resected specimen revealed $100 \%$ tumor necrosis and negative surgical margins. At the time of last follow-up (27 months postoperatively), no evidence of recurrence or metastasis was observed.

\section{Case 4}

A 7-year-old male patient was referred to our clinic with a limp due to left calf pain of 2 months, duration. He had no history of trauma. Mild swelling and tenderness were noticed on the anterior aspect of the proximal tibia. Radiographs of the left tibia revealed a lytic, expansile lesion with permeative 

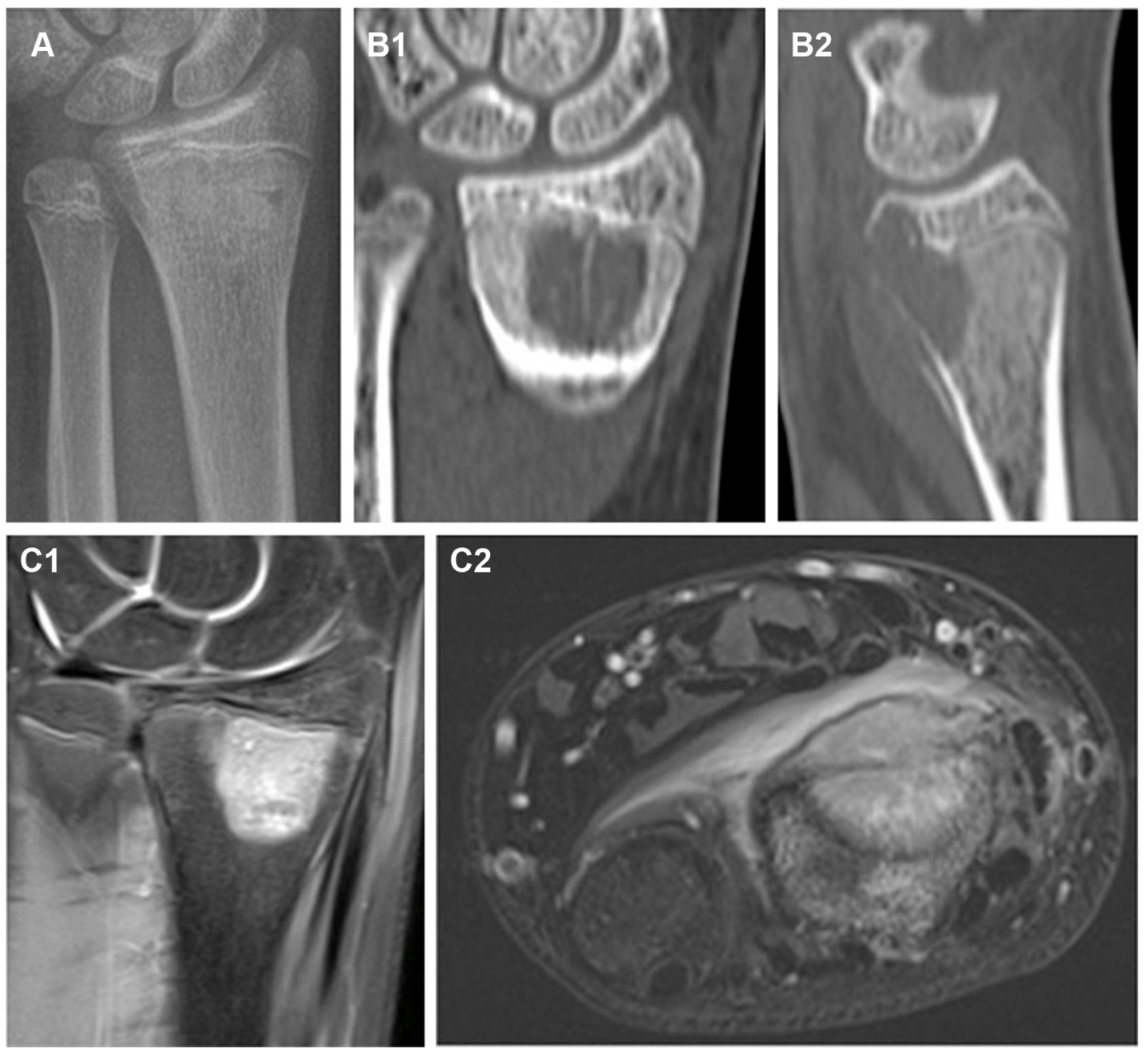

Figure 7 Preoperative images obtained by X-ray (A), CT (BI, B2), and MRI (CI, C2).

Note: Although X-ray is not suggestive for malignancy, eccentric, lytic, minimally expansile lesion with obvious periosteal reaction can be seen on CT images and MRI. Abbreviations: $\mathrm{CT}$, computed tomography; MRI, magnetic resonance imaging.

borders in the proximal diaphysis. A periosteal reaction was evident (Figure 9A1 and A2). MRI demonstrated peripheral contrast enhancement around a cystic-appearing lesion and marked bone marrow edema (Figure 9B1 and B2).

A Tru-Cut biopsy was performed through a cortical hole opened with a Jamshidi needle. The sections revealed small areas of lace-like osteoid with minimal cellular atypia. However, the amount of biopsy specimen was deemed inadequate to settle on or rule out a diagnosis of malignancy. Therefore, an open biopsy was performed, which unequivocally revealed the existence of atypical/immature osteoid and pleomorphic cells (Figure 10). A diagnosis of OBLOS was thus confirmed.

The patient underwent neoadjuvant chemotherapy. After completion of therapy, wide intercalary resection and reconstruction with combined cryopreserved massive autograft and vascularized fibular graft were performed. The necrosis rate was $90 \%$ in the resection specimen. At the time of last follow-up (26 months postoperatively), no evidence of recurrence or metastasis was documented.

\section{Discussion}

Osteoblastoma is a rare pathology, accounting for $\sim 1 \%$ of all bone tumors. ${ }^{6,7}$ OBLOS is also a rare form of osteosarcoma as shown by the fact that only four cases of OBLOS out of 330 osteosarcoma patients treated in our institution have been identified and reported in this study, which corresponds to $1.2 \%$ of all osteosarcoma cases in our series. Although this group of osteoblastic pathologies has a low incidence, their impact on orthopedic oncology is noteworthy because of the diagnostic difficulties they cause for clinicians and pathologists. ${ }^{8-10}$ Management of borderline osteoblastic tumors has been a subject of controversy for a long time. ${ }^{11}$ The borderline lesions have been rather hypothetically categorized as aggressive osteoblastomas, low-grade osteosarcomas, pseudosarcomatous osteoblastomas, and 

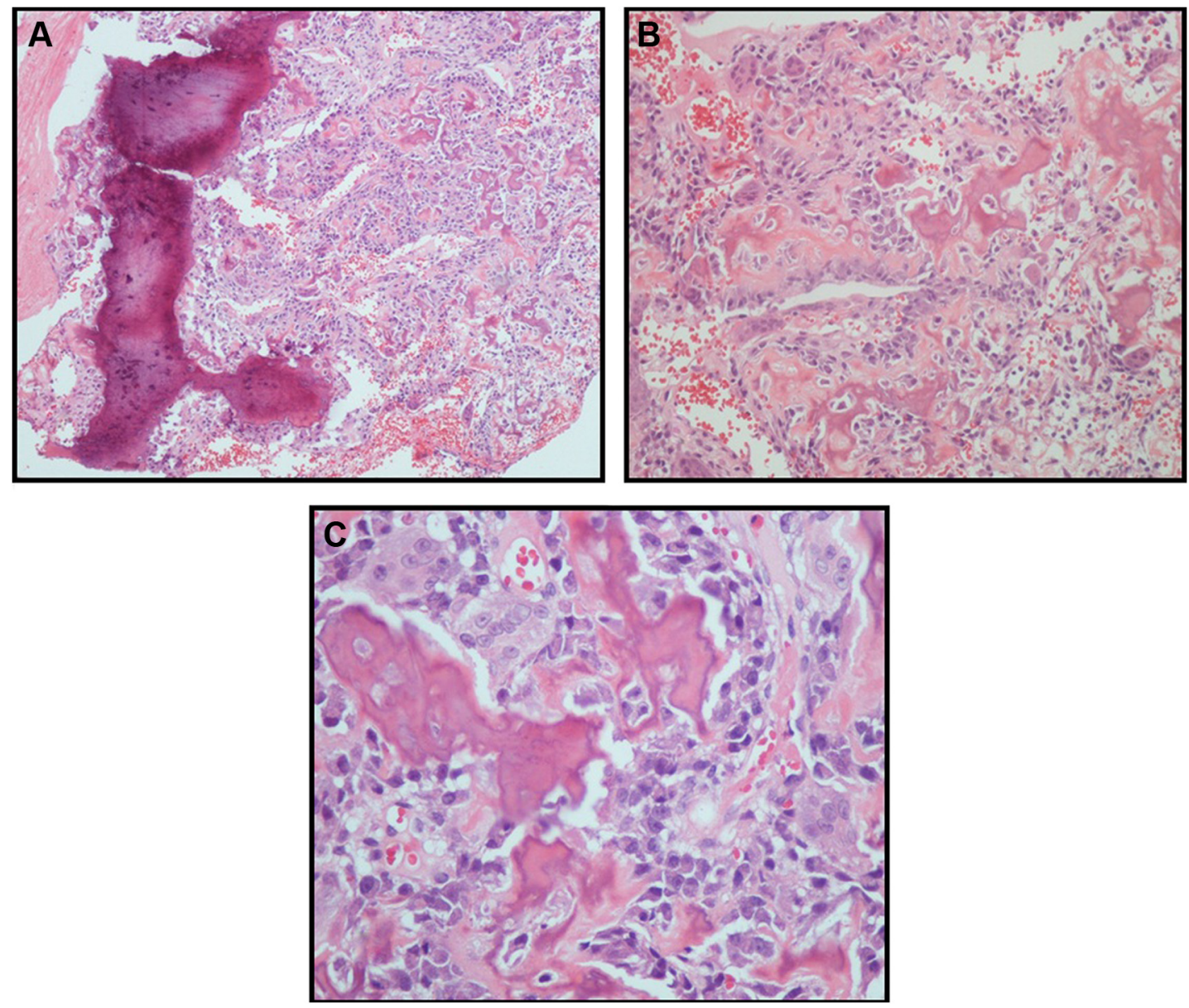

Figure 8 H\&E staining.

Notes: $($ A $) \times 100$, (B) $\times 200$, and $($ C) $\times 400$. (A) Low-power view of the tumor showing host bone entrapment, which is a manifestation of malignant behavior. (B and C) "Nidus"-like arrangement of osteoid, osteoblast-like cells, and tumoral cells.

Abbreviation: H\&E, hematoxylin and eosin.
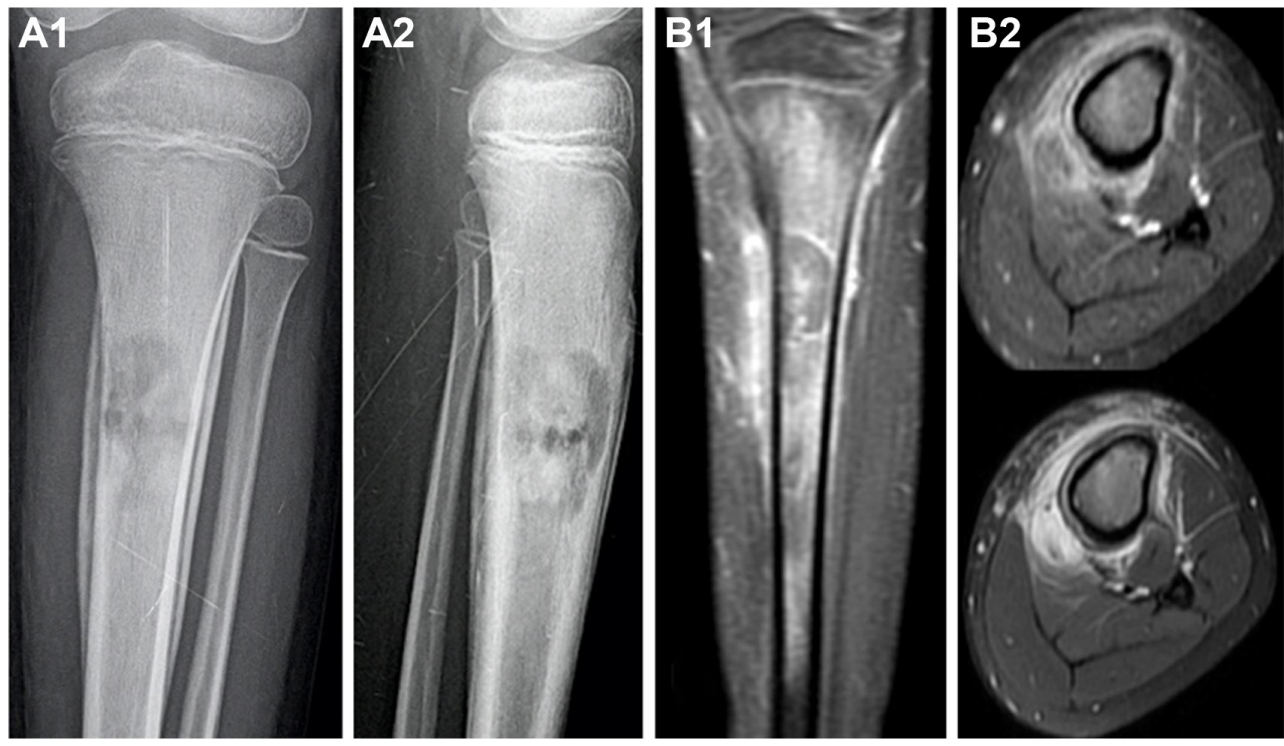

Figure 9 Preoperative images.

Notes: (AI, A2) X-rays (just after core needle biopsy) and (B I, B2) MRI.

Abbreviation: MRI, magnetic resonance imaging. 

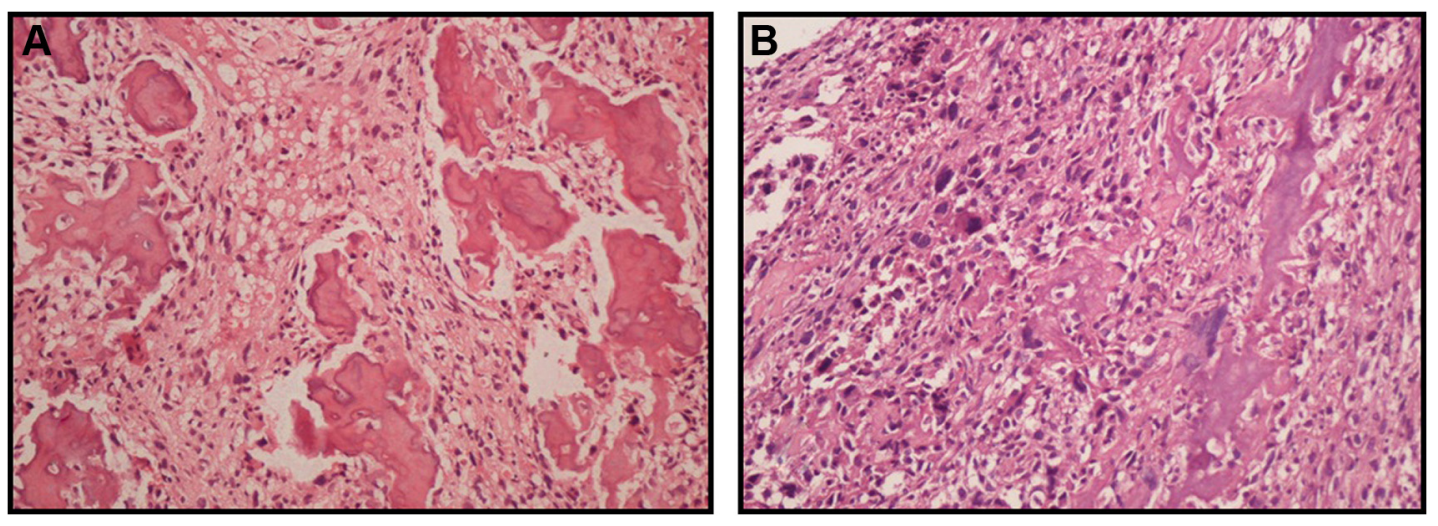

Figure 10 H\&E staining of open biopsy material.

Notes: $($ A $) \times 200$. Trabecular tumoral bone is observed. (B) $\times 200$. Striking pleomorphism and irregular osteoid formation are evident. Abbreviation: H\&E, hematoxylin and eosin

osteoblastomas, which transform into osteosarcomas. Multifocal osteoblastoma or osteoblastomatosis ${ }^{12}$ is another confusing entity, which is open to debate whether these are multiple occurring benign lesions, metachronous malignant lesions, or metastatic lesions.

Bertoni et $\mathrm{al}^{2,3}$ emphasized the importance of recognizing these controversial group of lesions as osteosarcomas in two consecutive studies in 1985 and 1993. In the latter study, cortical destruction and poorly defined borders were mentioned as suggestive of malignancy in radiographs, and tumor permeation of host bone was mentioned as the primary histological feature to differentiate OBLOS from osteoblastoma. Inadequate biopsy material was one of the leading causes for diagnostic inaccuracy. Initial biopsy was reported as benign in nine of eleven (82\%) patients in their 1993 study. This issue has come to our attention while evaluating our own patients since all four patients in our series were diagnosed as osteoblastoma in their initial biopsies performed with Tru-Cut needles. Actually, this problem was not associated with Tru-Cut needle's adequacy in sampling bone tumors but was totally related to larger than usual volume of tissue that cannot be harvested with Tru-Cut needle but is required to conveniently assess the histopathology in such critical and difficult cases. Therefore, it can be suggested that in the presence of suspicious radiographic characteristics and lack of substantial soft tissue component, Tru-Cut biopsy may be skipped and open biopsy might be performed straightaway.

The symptoms at presentation may not raise suspicion about such a specific disease and usually are not distinctive for osteoblastoma and osteosarcoma. However, progressive symptoms and new-onset clinical findings after an initial diagnosis of osteoblastoma must be alarming for the orthopedic oncologist in favor of malignancy.
The most common tumor locations were the tibia, the spine, the small bones of the skull, the face, and the foot in the two studies reported by Bertoni et al; 2,3 excluding the tibia, these are relatively uncommon locations for classical osteosarcoma. Even for the tibia cases, the tumor locations with regard to long axis of bone and radiographic appearances were not common for classical osteosarcoma. Although the tumor was located in the distal radius in two cases, proximal humerus (and also scapula) in one patient, and proximal tibia in one patient in our series, none of the cases demonstrated the typical radiographic appearance of an osteosarcoma. Therefore, OBLOS must always be considered in differential diagnoses of bone lesions in both axial and appendicular skeleton with atypical and aggressive appearance in radiographs.

OBLOS shares radiological features with osteoblastoma and osteosarcoma. The lesions often appear as lytic and expansile in radiographs. Irregular ossification or a sclerotic nidus may be observed inside a translucent lesion. Although the lesions are generally well-defined, a closer look at the radiographs reveals permeative borders and almost never a sclerotic rim. The cortex may be thinned with marked periosteal reaction, or obvious cortical destruction may be present. Rapidly growing lesions may show aneurysmal changes with pseudocystic appearance and peripheral contrast enhancement in the MRI. On the other hand, MRI may also demonstrate heterogenous contrast enhancement in the epicenter of the lesion and extensive bone marrow edema as evidenced by hyperintensity in T2-weighted images. Soft-tissue component is usually lacking in OBLOS lesions in contrast to classical osteosarcoma in MRI studies. In our series, radiological data at presentation were suggestive of malignancy for case 1 , case 3 , and case 4 , whereas 
Table I The radiological characteristics of classical OS, OB, and OBLOS

\begin{tabular}{|c|c|c|c|c|c|c|c|c|}
\hline $\begin{array}{l}\text { Type of } \\
\text { lesion }\end{array}$ & MRI-TI & MRI-T2 & $\begin{array}{l}\text { MRI } \\
\text { enhancement }\end{array}$ & CT & X-ray & $\begin{array}{l}\text { Periosteal } \\
\text { reaction }\end{array}$ & $\begin{array}{l}\text { Cortical } \\
\text { destruction }\end{array}$ & $\begin{array}{l}\text { Bone marrow, } \\
\text { soft-tissue edema }\end{array}$ \\
\hline $\mathrm{OB}$ & $\begin{array}{l}\text { Hypo- to } \\
\text { isointense }\end{array}$ & $\begin{array}{l}\text { Hypo- to } \\
\text { isointense }\end{array}$ & Permanent & $\begin{array}{l}\text { Lytic, ossification } \\
+/-, \text { with nidus }\end{array}$ & Lytic, well-defined & - & - & $+1-$ \\
\hline OS & $\begin{array}{l}\text { Hypointense } \\
\text { (hypointense } \\
\text { mineralized } \\
\text { component) }\end{array}$ & $\begin{array}{l}\text { Hyperintense } \\
\text { (hypointense } \\
\text { mineralized } \\
\text { component) }\end{array}$ & $\begin{array}{l}\text { Solid } \\
\text { component } \\
\text { enhancement }\end{array}$ & $\begin{array}{l}\text { Lytic and blastic } \\
\text { mixture, cortical } \\
\text { destruction }\end{array}$ & $\begin{array}{l}\text { Ill-defined, lytic, } \\
\text { and blastic }\end{array}$ & Aggressive & + & + \\
\hline OBLOS & Hypointense & $\begin{array}{l}\text { Intermediate } \\
\text { intensity }\end{array}$ & Heterogenous & $\begin{array}{l}\text { Well-defined/ } \\
\text { ill-defined, lytic, } \\
\text { sharply marginated }\end{array}$ & $\begin{array}{l}\text { Well-defined/ } \\
\text { ill-defined, lytic, } \\
\text { sharply marginated }\end{array}$ & $+1-$ & $+1-$ & $+1-$ \\
\hline
\end{tabular}

Note: Data from previous studies. ${ }^{1,6,8,9}$

Abbreviations: OS, osteosarcoma; OB, osteoblastoma; OBLOS, osteoblastoma-like osteosarcoma; MRI, magnetic resonance imaging; CT, computed tomography.

case 2 had a characteristic lesion resembling the nidus of osteoid osteoma or classical osteoblastoma. Consistent with our findings, the radiological appearance was typical of malignancy in only $64 \%$ of the 28 patients with OBLOS in the series described earlier. ${ }^{2,3}$ In addition, misdiagnosis of OBLOS as osteoid osteoma in one patient was also reported. ${ }^{3}$ Radiologic features of classical osteosarcoma, OBLOS, and osteoblastoma are summarized in Table 1 for a systematic approach to osteoblastic tumors.

Histologically, the major components of osteoblastoma are osteoblast-like cells, stromal cells, osteoclasts, osteoid tissue depositions, and blood vessels. In rare cases, destructive infiltration and cortical invasiveness (interruption) are evident. ${ }^{10}$ Aggressive osteoblastoma presents with high-level cellularity, nuclear atypia, immature tumorous bone (lace-like osteoid), and epithelioid osteoblasts. ${ }^{410}$ However, as seen in our first case, second biopsy revealed trabecular bone formation, osteoclastic giant cells, and prominent epithelioid osteoblasts that are characteristic for aggressive osteoblastoma. However, finally, the patient was diagnosed with OBLOS.

The morphology of OBLOS is variable. All features of aggressive osteoblastoma may be evident. Bertoni et $\mathrm{al}^{3}$ presented a series of 17 patients with OBLOS and concluded that two histological features were useful when seeking to differentiate OBLOS from osteoblastoma. OBLOS features permeation of surrounding host bone and lack of "maturation" toward the edges, whereas osteoblastoma tends to show peripheral maturation and is circumscribed. All osteoblastic tumors demonstrate osteoid formation in various patterns and amounts. Once the pathological diagnosis of OBLOS has been established with criteria such as pleomorphism and bone permeation, the "osteoblastoma-like" becomes only a descriptive tag for histological appearance and does not specify the tumor grade or aggressiveness (Table 2). The histological grade must be determined individually for each OBLOS case and treated accordingly. Chemotherapy is an essential adjuvant treatment modality in osteosarcoma and cannot be ignored with the assumption of all OBLOS cases having low-grade lesions.

Current literature lacks detailed information on survival analyses of OBLOS cases. Bertoni et $\mathrm{al}^{2,3}$ reported that seven of 17 cases $(41.2 \%)$ and two of eleven cases $(18.2 \%)$ died of disease in two patient series with OBLOS. However, in our series, all four cases were still alive and free of disease after a follow-up of 26 months, 23 months, 27 months, and 26 months, respectively.

These cases illustrate the importance of not hesitating to repeat the biopsy whenever suspicious bone lesions manifest aggressive behavior clinically and radiologically and also illustrate the challenges the pathologists will encounter when elements of benign and malignant etiologies coexist.

Table 2 Morphological differences between OBLOS, OB, and AOB

\begin{tabular}{|c|c|c|c|c|c|}
\hline $\begin{array}{l}\text { Type of } \\
\text { lesion }\end{array}$ & Cellularity & Cells & Osteoid production & $\begin{array}{l}\text { Atypical } \\
\text { mitosis }\end{array}$ & $\begin{array}{l}\text { Permeation of } \\
\text { adjacent bone }\end{array}$ \\
\hline OB & Low & $\begin{array}{l}\text { Osteoblast-like cells, } \\
\text { osteoclasts, stromal cells }\end{array}$ & $\begin{array}{l}\text { Trabecular organization of newly } \\
\text { formed osteoid }\end{array}$ & - & - \\
\hline $\mathrm{AOB}$ & High & Epithelioid osteoblasts & Lace-like osteoid & + & - \\
\hline OBLOS & Low-high & $\begin{array}{l}\text { Osteoblast-like cells, } \\
\text { osteoclasts, pleomorphism }\end{array}$ & $\begin{array}{l}\text { Random/trabecular organization of } \\
\text { newly formed osteoid; lace-like osteoid }\end{array}$ & + & + \\
\hline
\end{tabular}

Note: Data from previous studies. $19,11,13$

Abbreviations: OBLOS, osteoblastoma-like osteosarcoma; $O B$, osteoblastoma; $A O B$, aggressive osteosarcoma. 


\section{Conclusion}

When the radiographic appearance of a lesion is suggestive of malignancy, regardless of tumor location, it is wise to perform an open biopsy. In such a case, even if the pathological features are typical of osteoblastoma, the surgeon must be alert to tumor progression, recurrence, and/or metastasis. Clues in clinical behavior and radiological examinations must be carefully assessed to guide the pathologist. An accurate pathological diagnosis can be obtained through close cooperation between the orthopedic oncologist, musculoskeletal radiologist, and musculoskeletal pathologist.

\section{Disclosure}

The authors report no conflicts of interest in this work.

\section{References}

1. Hermann G, Klein MJ, Springfield D, Abdelwahab IF. Osteoblastoma like osteosarcoma. Clin Radiol. 2004;59(1):105-108.

2. Bertoni F, Bacchini P, Donati D, Martini A, Picci P, Campanacci M. Osteoblastoma-like osteosarcoma. The Rizzoli Institute experience. Mod Pathol. 1993;6(6):707-716.
3. Bertoni F, Unni KK, McLeod RA, Dahlin DC. Osteosarcoma resembling osteoblastoma. Cancer. 1985;55(2):416-426.

4. Khan L, Gupta MK, Singh PK, Agarwal A. Aggressive osteoblastoma of ilium: diagnosed on FNAC. Int J Case Rep Imag. 2012;3(8):34-38.

5. Xarchas KC, Leviet D. Osteoblastoma of the carpal scaphoid frequency and treatment. Acta Orthop Belg. 2002;68(5):532-536.

6. Murphey MD, Robbin MR, McRae GA, Flemming DJ, Temple HT, Kransdorf MJ. The many faces of osteosarcoma. Radiographics. 1997; 17(5):1205-1231.

7. Ottaviani G, Jaffe N. The epidemiology of osteosarcoma. Cancer Treat Res. 2009;152:3-13.

8. Steffner R. Benign bone tumors. Cancer Treat Res. 2014;162:31-63.

9. Kumar NL, Rosenberg AE, Raskin KA. Osteoblastoma-like osteosarcoma of the cuboid: a case report. J Orthop Surg Res. 2010;5:52.

10. Oliveira CR, Mendonça BB, Camargo OP, et al. Classical osteoblastoma, atypical osteoblastoma, and osteosarcoma: a comparative study based on clinical, histological, and biological parameters. Clinics (Sao Paulo). 2007;62(2):167-174.

11. Dorfman HD, Weiss SW. Borderline osteoblastic tumors: problems in the differential diagnosis of aggressive osteoblastoma and low grade osteosarcoma. Semin Diagn Pathol. 1984;1(3):215-234.

12. Kyriakos M, El-Khoury GY, McDonald DJ, et al. Osteoblastomatosis of bone. A benign, multifocal osteoblastic lesion, distinct from osteoid osteoma and osteoblastoma, radiologically simulating a vascular tumor. Skeletal Radiol. 2007;36(3):237-247.

13. Schajowicz F, Lemos C. Malignant osteoblastoma. J Bone Joint Surg. 1976;58(2):202-211.
Therapeutics and Clinical Risk Management

\section{Publish your work in this journal}

Therapeutics and Clinical Risk Management is an international, peerreviewed journal of clinical therapeutics and risk management, focusing on concise rapid reporting of clinical studies in all therapeutic areas, outcomes, safety, and programs for the effective, safe, and sustained use of medicines. This journal is indexed on PubMed Central, CAS,

\section{Dovepress}

EMBase, Scopus and the Elsevier Bibliographic databases. The manuscript management system is completely online and includes a very quick and fair peer-review system, which is all easy to use. Visit $\mathrm{http}: / /$ www.dovepress.com/testimonials.php to read real quotes from published authors. 